(79\%), employees (78\%), those in physical work (83\%), and in manufacturing (20\%), agriculture (17\%), and construction (16\%) sectors had the most claims. The oldest group, $65+$, had the highest proportion of claims in males (85\%), for the selfemployed (26\%), in sedentary work (21\%) and for the agriculture sector (23\%). Injuries to the face were common (30\%), particularly for workers aged $65+(56 \%)$, while back and hand/wrist injuries were also prevalent in those aged 55-59 and 60-64. Falls were prevalent (31\%) and highest in workers aged 55-59 (35\%) and 60-64 (33\%).

Conclusions The burden of work-related injuries in older workers will increase with their increasing participation in work. Interventions to protect older workers from injuries at work need to consider their specific characteristics and vulnerabilities to inform age-sensitive injury prevention strategies.

\section{RETURN TO WORK AFTER WORK INJURY: A COMPARATIVE POLICY EFFECTIVENESS STUDY}

'Alex Collie, 'Tyler Lane, 'Behrooz Hassani-Mahmooei, 'Jason Thompson, ${ }^{2}$ Christopher McLeod. 'Monash University, Australia; ${ }^{2}$ University of British Columbia, Canada

10.1136/injuryprev-2016-042156.194

Background Extended periods of time off work can have a negative impact on health. Australia has an array of state-based workers' compensation systems that seek to return injured workers to the workforce at minimal cost to society. These systems vary substantially in their design, and with respect to return to work (RTW) policy and practice. This study examined whether workers' compensation policy is an independent predictor of RTW following work injury.

Methods Comparative analyses of administrative data from eight Australian workers' compensation systems, containing 94,675 accepted work injury claims. Logistic regression controlling for demographic, work and injury factors were used to assess whether jurisdiction of claim had an independent impact on time loss from work at 4, 13, 26, 52 and 104 weeks post injury.

Results Substantial jurisdictional differences were identified at all time points post injury, after controlling for demographic, work and injury factors. Compared to New South Wales: workers in Victoria and South Australia had significantly greater odds of being off work (receiving income benefits) at all time points; workers in Tasmania had greater odds of being back at work (off benefits) at all time points, while RTW of workers in Western Australia and Queensland improved at later time points. The magnitude of jurisdiction effects were as or more substantial than that identified for injury type, age, gender, occupation and socioeconomic status.

Conclusions Workers' compensation system design has a significant and independent impact on RTW following work injury. Findings reveal the need for identification and implementation of policies and practices that promote timely and appropriate RTW.

\section{NURSES AND ANTINEOPLASTIC AGENTS: FACTORS INFLUENCING EXPOSURE RISK}

'David M DeJoy, ${ }^{2}$ Todd D Smith, ${ }^{1}$ Henok Woldu, ${ }^{3}$ Aimee Dyal. ${ }^{1}$ University of Georgia, U.S. $A_{;}{ }^{2}$ Indiana University, U.S.A; ${ }^{3}$ Kennesaw State University, U.S.A
Background Chemotherapy drugs pose a hazard to those administering them. This study examined the effects of individual, situational, and organisational factors on compliance with personal protective equipment (PPE), use of engineering controls, and exposures in a sample of oncology nurses $(n=1.915)$.

Methods Data came from the web-based NIOSH Health and Safety Practices Survey of Healthcare Workers. Survey measures included demographics, employment situation, safety practices, training, safety climate, antineoplastic drug (AD) administration, and exposures.

Results Preliminary descriptive and bivariate analyses were conducted. Two stepwise multiple regressions were computed with PPE compliance and engineering controls as outcome variables. For PPE, safety climate and familiarity with guidelines were the strongest predictors of use (both $\mathrm{p}<0.0001)$. Non-profit status, hospital setting, and having more employees were also relatively strong predictors of compliance ( $p<0.002$ to 0.006 ). The strongest predictors for engineering controls were relevant policies/ procedures, safety climate, and familiarity with guidelines (all $p<0.0001)$. Engineering control practices were also better for those in non-profit $(\mathrm{p}<0.001)$ and government settings ( $\mathrm{p}<0.007$ ). A final stepwise multiple logistic model assessed occupational exposures (skin contact and/or spill/leak of AD). Exposure risk increased with number of $\mathrm{AD}$ administrations ( $p<0.0001$ ), while the use of engineering controls reduced exposures by nearly 30\% ( $\mathrm{p}<0.0001$ ). Safety policies/procedures, PPE and safety climate also reduced exposures.

Conclusions This study highlights the importance of organisational and safety management practices in preventing exposures to antineoplastic agents. Exposures increased with the number of AD administrations, but policies/procedures, associated controls, and safety climate reduced risk.

\section{MENTAL FIRST AID MODEL HELPS WORK COMMUNITY IN VARIOUS DIFFICULT SITUATIONS}

${ }^{1}$ Hanna Jurvansuu, ${ }^{1}$ Marja Paukkonen, ${ }^{2}$ Anna-Maria Teperi. ${ }^{1}$ Education Department, City of Helsinki, Finland; ${ }^{2}$ Finnish Institute of Occupational Health

\subsection{6/injuryprev-2016-042156.196}

Background In the city of Helsinki, employees report thousands of threatening situations at work every year. Two thirds of victims typically cope on their own after a critical incident but one third needs help to manage. If not handled shortly, incidents may cause excessive stress or absence from work. Occupational health care organises debriefing after severe incidents but many minor incidents are left unhandled.

Description of the problem There was a need for mental first aid" at workplaces, so that personnel facing incidents could unload the mental burden right away. How to handle incidents quickly, during the workday, with a colleague? After the incident, the manager is usually responsible for examining the facts whereas colleagues could support with the mental process.

Results A short training based on Critical Incident Stress Management (CISM) was organised for some employees and leaders. The training included e.g. lecture and discussion of safety culture at work and of human reactions during and after incidents. A discussion model of mental first aid (in Finnish hetipurku) and practical tools to support it were presented and practiced. According to the participants' experience, the model turned out to be useful 